\title{
Immunogenicity and safety of a 12-valent pneumococcal conjugate vaccine in infants aged 6-10 weeks: a randomized double-blind active-controlled trial
}

Jonghoon Shin, BS ${ }^{1}$, Jamaree Teeratakulpisarn, $\mathrm{MD}^{2}$, Thanyawee Puthanakit, $\mathrm{MD}^{3}$, Tuangtip Theerawit, BSN ${ }^{3}$, Ji Hwa Ryu, $\mathrm{MS}^{1}$, Jinhwan Shin, $\mathrm{MS}^{1}$, Seulgi Lee, BS${ }^{1}$, Hayoung Lee, BS ${ }^{1}$, Kyungjun An, MS ${ }^{1}$, Hun Kim, PhD ${ }^{1}$

${ }^{1}$ SK bioscience, Seongnam, Korea; ${ }^{2}$ Department of Pediatrics, Khon Kaen University, Khon Kaen, Thailand; ${ }^{3}$ Department of Pediatrics and Center of Excellence in Pediatric Infectious Diseases and Vaccine Faculty of Medicine, Chulalongkorn University, Bangkok, Thailand

Background: Pneumococcal diseases among children aged $<5$ years worldwide are associated with high annual mortality rates.

Purpose: This study aimed to evaluate the immunogenicity and safety of GBP411, a 12-valent pneumococcal conjugant vaccine, with a dosing schedule of 2 primary doses plus 1 booster dose $(2 p+1)$ in healthy infants.

Methods: This randomized active-controlled (Prevnar 13) double-blind phase 2 trial enrolled healthy subjects aged 6-10 weeks. Three serum concentrations of pneumococcal serotypespecific immunoglobulin $\mathrm{G}(\mathrm{IgG})$ were evaluated using the pneumococcal serotype-specific pneumonia polysaccharide enzyme-linked immunosorbent assay at 1 month after the primary doses and before and 1 month after the booster dose. The pneumococcal serotype-specific IgG titer was evaluated using a multiplex opsonophagocytic assay in a subset of 15 subjects per group.

Results: After administration of the primary doses, the proportion of subjects who achieved pneumococcal serotypespecific $\operatorname{IgG}$ concentrations of $>0.35 \mu \mathrm{g} / \mathrm{mL}$ was lower for some serotypes in the GBP411 group than in the comparator group (6B: $20.83 \%$ vs. $39.22 \%, P=0.047$ and $19 \mathrm{~A}: 58.33 \%$ vs. $90.20 \%, P<0.001)$. However, after administration of the booster dose, $>97 \%$ of the subjects in each group achieved IgG concentrations of $\geq 0.35 \mu \mathrm{g} / \mathrm{mL}$ for all 12 serotypes. Increased immunogenicity was observed for some serotypes that showed significant intergroup differences after administration of the primary doses but not after the booster dose. We also found no significant intergroup difference in the overall incidence of solicited local adverse events. Furthermore, the overall incidence of solicited systemic adverse events was significantly lower in the GBP411 group than in the comparator vaccine group (79.59\% vs. $98.04 \% ; P=0.003$ ).

Conclusion: The GBP411 vaccine with a dosing schedule of $2 p+1$ may be immunogenic and safe for healthy infants.
Key words: 12-Valent pneumococcal conjugate vaccine, Streptococcus pneumoniae, Immunogenicity, Safety, Infant

\section{Key message}

Question: The immunogenicity and safety of GBP411 when administered to healthy infants are not understood.

Finding: The intergroup differences were not significant for all 12 serotypes after the booster dose. The overall incidence of solicited local adverse events between the groups did not differ significantly.

Meaning: GBP411 with a 2p+1 dosing schedule induced a substantial immune response, and may be safe for administration to healthy infants.

\section{Introduction}

Pneumococcal diseases caused by Streptococcus pneumoniae (S. pneumoniae or pneumococcus), including pneumonia, meningitis, and bacteremia, result in deaths in approximately half a million children under the age of 5 years globally every year, with a particularly high number of deaths occurring in Africa and Asia. ${ }^{1,2)}$ Infants and toddlers aged $\leq 2$ years who have been the least immunogenic to the related vaccines are the most vulnerable, with the incidence of invasive pneumococcal diseases (IPDs) being the highest. ${ }^{3}$ The global number of deaths in children aged 1-59 months was estimated to be 318,000 (uncertainty range, 207,000-395,000) in 2015; pneumonia accounted for $81 \%$ of the deaths $(257,000$; uncertainty range, $182,000-268,000) .^{2)}$

The pneumococcal conjugate vaccine (PCV) was introduced for use in infants in the year 2000, and 2 kinds of PCV (10-valent Synflorix, GlaxoSmithKline, Brentford, UK; 13-valent Prevnar 13, Pfizer, New York, USA) are currently marketed worldwide.

\footnotetext{
Corresponding author: Hun Kim, PhD. SK bioscience, Eco Hub, 332, Pangyo-ro, Bundang-gu, Seongnam 13493, Korea 凶E-mail: ebolakim@sk.com, https://orcid.org/0000-0003-3726-7094

Received: 6 September, 2019, Revised: 21 November, 2019, Accepted: 22 November, 2019

This is an open-access article distributed under the terms of the Creative Commons Attribution Non-Commercial License (http://creativecommons.org/licenses/bync/4.0/) which permits unrestricted non-commercial use, distribution, and reproduction in any medium, provided the original work is properly cited. Copyright (c) 2020 by The Korean Pediatric Society
} 


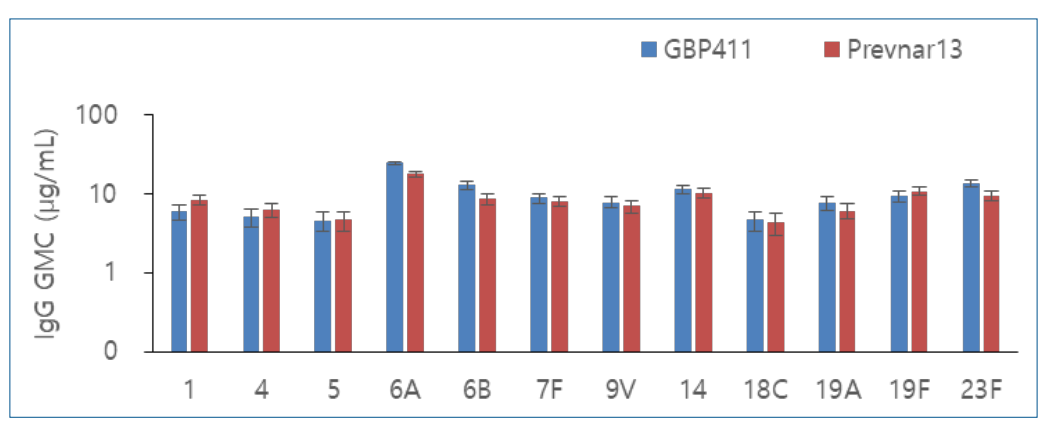

Graphical abstract. Comparison of pneumococcal immunoglobulin $\mathrm{G}(\mathrm{lgG})$ geometric mean concentrations (GMCs) after booster dose.

1,3) These vaccines had been approved for a 4-dosing regimen (3 primary doses with 1 booster [3p+1]). However, the World Health Organization (WHO) recommends a 3 dosing regimen, i.e., either as 2 primary doses with 1 booster $(2 p+1)$ or 3 primary doses without a booster $(3 p+0)$ rather than $3 p+1$, because this is the most practical dosing schedule for infants. ${ }^{4}$ Actually, many countries use the $2 p+1$ dosing schedule as part of their national immunization program, such as the United Kingdom, Singapore, and Belgium. It has been reported that the $3 p+1$ schedule elicits better immunogenicity for some serotypes than that elicited by the $2 p+1$ dosing schedule after the primary doses. Nevertheless, responses for all serotypes have been found comparable after the booster dose for both dosing regimens. ${ }^{5)}$ With regard to the 3 dosing regimen, it is known that compared to a $3 p+0$ schedule, the $2 p+1$ schedule results in higher antibody geometric mean concentrations (GMCs) and is associated with a similar or higher percentage of responders because of the booster dose, which may provide longer protection and have greater indirect effects. ${ }^{6}$ SK bioscience has developed a 12-valent pneumococcal conjugate vaccine (GBP411) that meets the needs of developing countries (e.g., in terms of serotype composition and vaccine presentation). Compared to Prevnar 13, GBP411 is a 12-valent PCV (serotype 1, 4, 5, 6A, 6B, 7F, 9V, 14, 18C, 19A, 19F, and 23F) excluding serotype 3 , which has been reported to have a limited protective effect on the incidence of IPD. ${ }^{6,7)}$ In both vaccines, nontoxic diphtheria toxin molecule (CRM197) is used as a carrier protein but different polysorbates are used as suspending agents. Each $0.5 \mathrm{~mL}$ dose of GBP411 contains approximately $2.2 \mu \mathrm{g}$ of each of all the containing S. pneumoniae serotypes except $4.4 \mu \mathrm{g}$ of $6 \mathrm{~B}$ saccharides. This study was designed to evaluate the immunogenicity and safety of GBP411 with $2 p+1$ dosing schedule in comparison to those of Prevnar 13 when administered to healthy infants.

\section{Methods}

\section{Study design}

This was a randomized, active-controlled, double-blind, phase 2 trial conducted at 2 sites in Thailand. The trial was conducted in accordance with ethical principles that had their origin in the Declaration of Helsinki. The protocol was reviewed and approved by the responsible ethics committee (Khon Kaen University IRB No. 05/2560, Chulalongkorn University IRB No. 725/59). Written informed consent was obtained from all parents or legal authorized representatives prior to enrolling the subjects in the study. A total of 3 doses of the GBP411 or comparator vaccine (Prevnar 13) were administered to the subjects at 6 to 10 weeks after birth, 8 to 10 weeks after the first dose (primary doses), and 39 to 52 weeks after birth (booster dose). Blood sam. ples were collected at 4 to 6 weeks after the primary doses and before and after the booster dose. The primary objective was to evaluate the immunogenicity and secondary objective was to evaluate the safety of GBP411 when administered to healthy infants.

\section{Study population}

Subjects were eligible if they were aged 6-10 weeks, had been vaccinated against hepatitis $B$ at birth, and were healthy. Subjects were ineligible if they had been previously vaccinated with other vaccines except for the bacillus Calmette-Guerin vaccine within 4 weeks after birth and hepatitis $B$ vaccine at birth, if vaccination was contraindicated, had known or suspected immune deficiency or suppression, had a history of a disease caused by S. pneumoniae or Haemophilus influenzae type b (Hib), had a severe chronic disorder including a congenital malformation or had a history of significant neurological diseases or seizures, had received blood products or immunoglobulins, and had received immunosuppressants or immunomodulators. Eligible subjects were randomly allocated in a 1:1 ratio to receive GBP411 or comparator vaccine by using a permuted block randomization schedule.

\section{Interventions}

The subjects were administered each dose of GBP411 or comparator vaccine intramuscularly in the anterolateral region of the thigh. GBP411 contains 12 serotypes from pneumococcal serotypes 1, 4, 5, 6A, 6B, 7F, 9V, 14, 18C, 19A, 19F, and 23F, which are identical to those in the comparator vaccine (Prevnar 13), except for serotype 3. GBP411 is available as a 2-dose 1.0$\mathrm{mL}$ vial. Exactly $0.5 \mathrm{~mL}$ of GBP411 was used from the 2-dose $1.0-\mathrm{mL}$ vial for injection. The comparator vaccine, which is available as a $0.5-\mathrm{mL}$ prefilled syringe, was used for injection directly. The study vaccines were dispensed and administered by 
unblinded study staff members who were not involved in subsequent participant assessments. All other study staff members and the participants were blinded to the vaccine administered. Concomitant vaccines such as DTaP (diphtheria, tetanus, and pertussis) vaccine, inactivated polio vaccine, Hib conjugate vaccine, and hepatitis B vaccine were allowed to be administered opposite the administration site of the investigational product on the same day as the study vaccine while the primary doses were administered.

\section{Immunogenicity assessments}

The immunogenicity of the study vaccine was assessed based on serum concentrations of pneumococcal serotype-specific immunoglobulin $G$ (IgG) by using a pneumococcal serotypespecific pneumonia polysaccharide enzyme-linked immunosorbent assay (ELISA). In a subset of 15 subjects randomly extracted per group, the pneumococcal serotype-specific IgG antibody titer was evaluated using a multiplex opsonophagocytic assay (MOPA). The primary endpoint was the proportion of subjects with pneumococcal serotype-specific IgG antibody concentrations of $\geq 0.35 \mu \mathrm{g} / \mathrm{mL}$ after the primary doses. The secondary endpoints were (1) pneumococcal serotype-specific IgG GMC after the primary doses and before the booster dose; (2) proportion of subjects with pneumococcal serotype specific IgG antibody concentrations of $\geq 0.35 \mu \mathrm{g} / \mathrm{mL}$ after the booster dose; (3) pneumococcal serotype-specific IgG GMC after the booster dose; and (4) pneumococcal serotype-specific MOPA antibody titer of $\geq 1: 8$ in a subset of 15 subjects per group after the primary doses and after the booster dose. All immunogenicity assessments were performed at the laboratory of SK bioscience located in Republic of Korea.

\section{Safety assessments}

Adverse events (AEs) were collected using subjects' diaries retrieved at regular visits after each vaccination and medical examinations at regular visits during the treatment period based on interviews conducted by the principal investigator or subinvestigator, as well as through spontaneous reporting by legally authorized representatives, including parents, at any time. AEs were regularly monitored to obtain additional safety information until event resolution or until follow-up was no longer possible. The severity of the solicited local and systemic AEs were graded on a scale of 1 to 4 based on the guidelines of the Korean Ministry of Food and Drug Safety and U.S. Food and Drug Administration (US FDA) ${ }^{8)}$ The endpoint of the safety assessment was the incidence of solicited local and systemic AEs for 7 days after each vaccination and the incidence of unsolicited AEs throughout the study until last subject's last visit. Serious AEs were collected and followed for 6 months after the subject's last vaccination even if the subject was discontinued or withdrawn.

\section{Statistical analysis}

The demographic information for all subjects enrolled in this study (intention-to-treat set) was analyzed, and descriptive statistics including the mean and standard deviation values are presented. Statistical analyses of immunogenicity assessment endpoints were performed for the per protocol set (PP set, defined as all subjects who completed the study procedures without major protocol violations) as the primary analysis set as well as for the full analysis set (FA set, defined as all randomized subjects who had all 3 doses of the study vaccine and were assessed for immunogenicity after administration). Furthermore, safety endpoints in the safety set (defined as all subjects who were enrolled in the study and received vaccination) were statistically analyzed.

For the immunogenicity endpoint, the proportion of subjects with pneumococcal serotype-specific IgG antibody concentrations of $\geq 0.35 \mu \mathrm{g} / \mathrm{mL}$ or pneumococcal serotype-specific MOPA antibody titer of $\geq 1: 8$ was calculated for each treatment group along with the $95 \%$ confidence intervals (CIs) for the proportions. The $95 \%$ CI for the proportion difference was determined using the Wald method, and the $P$ value for the proportion difference was determined using the chi-square or Fisher exact test. The calculation for pneumococcal serotypespecific IgG GMC and MOPA geometric mean titer (GMT) was performed by considering the anti-log 10 of the arithmetic mean of the $\log$ (base 10) transformed, and 95\% CI was derived using $t$ distribution. The $P$ value for the mean difference was determined using the 2-sample t test.

For the safety endpoint, the proportion of subjects with solicited AEs and unsolicited AEs was calculated for each treatment group along with the $95 \%$ CIs for the proportions. The $P$ value for the proportion difference was determined using the chi-square test. $P<0.05$ was considered statistically significant. Because this was an exploratory trial, no statistical hypothesis was tested.

\section{Results}

\section{Baseline characteristics and disposition of the subjects}

From May 2017 to June 2017, 102 subjects were screened. Of them, 100 eligible subjects were randomly assigned to the GBP411 or comparator vaccine groups at a 1:1 ratio (49 subjects were randomized to receive the GBP411 and 51 to receive the comparator vaccine). A total of 100 subjects were included in the safety set, of whom 99 were included in the FA and PP sets. Informed consent for study participation was withdrawn for 1 subject and this subject was excluded from the FA and PP sets. The subjects' disposition is shown in Fig. 1. Demographic characteristics for all subjects are summarized in Table 1 . There were no statistically significant differences in demographic characteristics or medical history between the 2 groups.

\section{Immunogenicity results}

For 10 of the 12 serotypes $(1,4,5,6 \mathrm{~A}, 7 \mathrm{~F}, 9 \mathrm{~V}, 14,18 \mathrm{C}, 19 \mathrm{~F}$, and 23F), intergroup differences in the proportion of subjects with pneumococcal serotype-specific IgG antibody concentrations of 
$\geq 0.35 \mu \mathrm{g} / \mathrm{mL}$ after the primary doses were not significant. For serotypes $6 \mathrm{~B}$ and $19 \mathrm{~A}$, the proportion of subjects meeting the criterion was significantly lower in the GBP411 group than in the comparator vaccine group $(20.83 \%$ vs. $39.22 \%, P=0.047$ and $58.33 \%$ vs. $90.20 \%, P<0.001$, respectively). After the administration of the booster dose, however, $>97 \%$ of the subjects in each group had pneumococcal serotype-specific IgG antibody

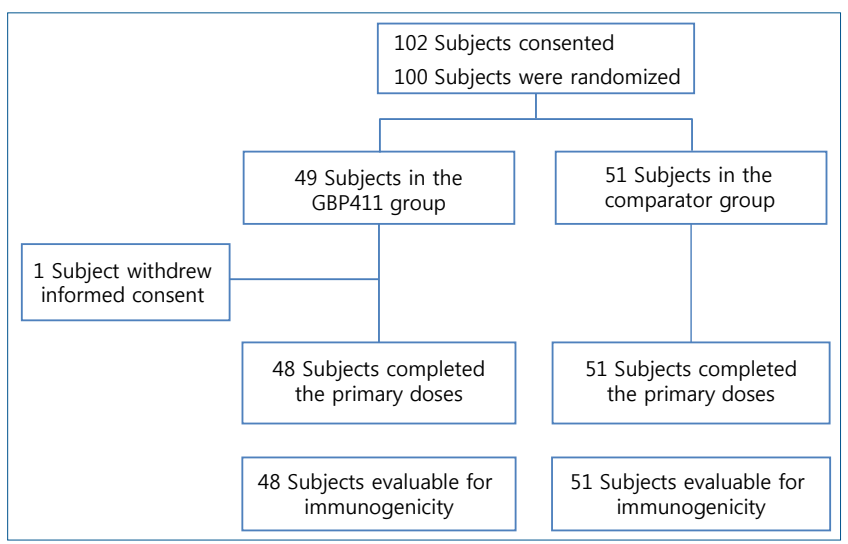

Fig. 1. Flowchart of the subject enrollment process. concentrations that reached $\geq 0.35 \mu \mathrm{g} / \mathrm{mL}$ for all 12 serotypes (Table 2).

For the GMCs, no significant difference was detected between the groups for 7 of the 12 serotypes (4, 7F, 9V, 14, 18C, 19F, and $23 \mathrm{~F})$ throughout the study. Although the GMCs for serotypes $1,5,6 \mathrm{~A}, 6 \mathrm{~B}$, and 19A in the GBP411 group were significantly lower than those in the comparator vaccine group $(1: P<0.001$; 5: $P=0.019$; 6A: $P=0.024$; 6B: $P=0.007$; 19A: $P \leq 0.001)$ after the primary doses, the GMCs for all 12 serotypes were comparable between the groups after the booster dose was administered (Table 3).

In the subset of 15 subjects per group for whom MOPA evaluations were performed, the proportion of subjects with MOPA titer of $\geq 1: 8$ after the primary doses ranged from $73.33 \%$ to $100 \%$ and was comparable between the 2 groups. There was no significant difference with regard to the 12 serotypes between the groups. After the booster dose, all subjects had a MOPA antibody titer of $\geq 1: 8$ for the 12 serotypes (Table 4). In case of GMTs, no statistical difference was noted between the groups for 10 of the 12 serotypes $(1,4,6 \mathrm{~A}, 6 \mathrm{~B}, 9 \mathrm{~V}, 14,18 \mathrm{C}, 19 \mathrm{~A}, 19 \mathrm{~F}$, and $23 \mathrm{~F}$ ) after the primary doses. The GMT for serotype 5 was significantly lower in the GBP411 group $(P=0.040)$, whereas

Table 1. Baseline characteristics of the study subjects

\begin{tabular}{|c|c|c|c|c|}
\hline Variable & GBP411 $(n=49)$ & Prevnar $13(n=51)$ & Total $(n=100)$ & $P$ value \\
\hline Age (day) & $54.86 \pm 5.05$ & $56.24 \pm 5.31$ & $55.56 \pm 5.20$ & 0.187 \\
\hline Gestational period (wk) & $38.51 \pm 1.08$ & $38.41 \pm 0.92$ & $38.46 \pm 1.00$ & 0.625 \\
\hline Sex & & & & $0.395^{c)}$ \\
\hline Male & $18(36.73)$ & $23(45.10)$ & $41(41.00)$ & \\
\hline Female & $31(63.27)$ & $28(54.90)$ & $59(59.00)$ & \\
\hline Height $(\mathrm{cm})$ & $55.93 \pm 1.46$ & $55.56 \pm 2.16$ & $55.74 \pm 1.85$ & 0.311 \\
\hline Weight (kg) & $5.08 \pm 0.50$ & $4.95 \pm 0.55$ & $5.02 \pm 0.53$ & 0.247 \\
\hline Tympanic temperature $\left({ }^{\circ} \mathrm{C}\right)$ & $36.68 \pm 0.31$ & $36.66 \pm 0.33$ & $36.67 \pm 0.32$ & 0.769 \\
\hline
\end{tabular}

Values are presented as mean \pm standard deviation or number (\%).

a) Age (day)=date of informed consent-date of birth. ${ }^{b} P$ values were based on a 2 -sample $t$ test for continuous data. ${ }^{c}$ Chi-square test for categorical data.

Table 2. Proportions of subjects who achieved a pneumococcal immunoglobulin G antibody concentration of $\geq 0.35 \mu \mathrm{g} / \mathrm{mL}$

\begin{tabular}{|c|c|c|c|c|c|c|c|c|c|c|}
\hline \multirow[b]{2}{*}{ Serotype } & \multicolumn{5}{|c|}{ After the primary dose } & \multicolumn{5}{|c|}{ After the booster dose } \\
\hline & $\begin{array}{c}\text { GBP411 } \\
(n=48)\end{array}$ & $\begin{array}{l}\text { Prevnar } 13 \\
\quad(n=51)\end{array}$ & Difference & $95 \% \mathrm{Cl}^{\mathrm{a})}$ & $P$ value $^{\mathrm{b})}$ & $\begin{array}{c}\text { GBP411 } \\
(n=48)\end{array}$ & $\begin{array}{l}\text { Prevnar } 13 \\
\quad(n=51)\end{array}$ & Difference & $95 \% \mathrm{Cl}^{\mathrm{a})}$ & $P$ value ${ }^{\text {b) }}$ \\
\hline 1 & 91.67 & 98.04 & -6.37 & (-15.07 to 2.33$)$ & $0.196^{d)}$ & 100.00 & 100.00 & 0.00 & $(0.00-0.00)$ & - \\
\hline 4 & 97.92 & 98.04 & -0.12 & $(-5.68$ to 5.43$)$ & $1.000^{d)}$ & 100.00 & 100.00 & 0.00 & $(0.00-0.00)$ & - \\
\hline 5 & 93.75 & 94.12 & -0.37 & (-9.79 to 9.05$)$ & $1.000^{\mathrm{d})}$ & 100.00 & 100.00 & 0.00 & $(0.00-0.00)$ & - \\
\hline $6 \mathrm{~A}$ & 85.42 & 94.12 & -8.70 & (-20.60 to 3.20$)$ & $0.192^{\mathrm{d})}$ & 100.00 & 100.00 & 0.00 & $(0.00-0.00)$ & - \\
\hline $6 \mathrm{~B}$ & 20.83 & 39.22 & -18.38 & $(-36.04$ to -0.74$)$ & $0.047^{c)}$ & 97.92 & 100.00 & -2.08 & $(-6.12$ to 1.96$)$ & $0.485^{d)}$ \\
\hline $7 F$ & 100.00 & 100.00 & 0.00 & $(0.00-0.00)$ & - & 100.00 & 100.00 & 0.00 & $(0.00-0.00)$ & - \\
\hline $9 \mathrm{~V}$ & 95.83 & 96.08 & -0.25 & (-8.02 to 7.53$)$ & $1.000^{\mathrm{d})}$ & 100.00 & 100.00 & 0.00 & $(0.00-0.00)$ & - \\
\hline 14 & 95.83 & 98.04 & -2.21 & $(-9.03$ to 4.61$)$ & $0.610^{d)}$ & 100.00 & 98.04 & 1.96 & $(-1.84$ to 5.77$)$ & $1.000^{\mathrm{d})}$ \\
\hline $18 \mathrm{C}$ & 97.92 & 96.08 & 1.84 & $(-4.85$ to 8.53$)$ & $1.000^{d)}$ & 100.00 & 100.00 & 0.00 & $(0.00-0.00)$ & - \\
\hline $19 \mathrm{~A}$ & 58.33 & 90.20 & -31.86 & $(-48.03$ to -15.71$)$ & $<0.001^{c)}$ & 97.92 & 100.00 & -2.08 & $(-6.12$ to 1.96$)$ & $0.485^{d)}$ \\
\hline $19 F$ & 100.00 & 98.04 & 1.96 & $(-1.85$ to 5.77$)$ & $1.000^{d)}$ & 97.92 & 100.00 & -2.08 & $(-6.12$ to 1.96$)$ & $0.485^{d)}$ \\
\hline $23 \mathrm{~F}$ & 70.83 & 78.43 & -7.60 & (-24.71 to 9.52$)$ & $0.385^{c)}$ & 100.00 & 100.00 & 0.00 & $(0.00-0.00)$ & - \\
\hline
\end{tabular}

$\mathrm{Cl}$, confidence interval.

a) $95 \%$ Cls for the differences in proportions between the treatment groups were determined using the Wald method. ${ }^{\text {b) }} P$ value for the proportion was determined using ${ }^{\mathrm{c})}$ the chi-square test or ${ }^{\mathrm{d})}$ Fisher exact test.

Boldface indicates a statistically significant difference with $P<0.05$. 
Table 3. Geometric mean concentrations of pneumococcal immunoglobulin G

\begin{tabular}{|c|c|c|c|c|c|c|c|c|c|c|c|c|c|c|c|}
\hline \multirow{2}{*}{$\begin{array}{l}\text { Sero- } \\
\text { type }\end{array}$} & \multicolumn{5}{|c|}{ After the primary dose } & \multicolumn{5}{|c|}{ Before the booster dose } & \multicolumn{5}{|c|}{ After the booster dose } \\
\hline & $\begin{array}{l}\text { GBP411 } \\
(n=48)\end{array}$ & $\begin{array}{l}\text { Prevnar } 13 \\
(n=51)\end{array}$ & Ratio & $95 \% \mathrm{Cl}^{\mathrm{a})}$ & $\begin{array}{c}P \\
\text { value }^{\mathrm{b})}\end{array}$ & $\begin{array}{c}\text { GBP411 } \\
(n=48)\end{array}$ & $\begin{array}{l}\text { Prevnar } 13 \\
(n=51)\end{array}$ & Ratio & $95 \% \mathrm{Cl}^{\mathrm{a})}$ & $\begin{array}{c}P \\
\text { value }^{\mathrm{b})}\end{array}$ & $\begin{array}{c}\text { GBP411 } \\
(n=48)\end{array}$ & $\begin{array}{l}\text { Prevnar } 13 \\
(n=51)\end{array}$ & Ratio & $95 \% \mathrm{Cl}^{a)}$ & $\begin{array}{c}P \\
\text { value }^{b}\end{array}$ \\
\hline 1 & 1.54 & 33 & 0.46 & $(0.32-($ & $<0.001$ & 0.52 & .00 & 0.52 & $(0.36-0.76)$ & $<0.001$ & 5.95 & .40 & 0.71 & 04) & 0.073 \\
\hline 4 & 3.04 & 15 & 0.97 & $(0.72-1.30)$ & 0.817 & 0.78 & 0.81 & 0.97 & $(0.7$ & 0.836 & 5.11 & 28 & 0.81 & .15) & 0.237 \\
\hline 5 & 1.10 & 73 & 0.64 & $(0.44-$ & 0.019 & 0.96 & 0.90 & 1.07 & $(0.7$ & 0.717 & 4.62 & 67 & .99 & 36) & 0.942 \\
\hline $6 \mathrm{~A}$ & 1.44 & 2.47 & 0.58 & $(0.36-0.93)$ & 0.024 & 1.59 & 1.55 & 1.02 & $(0.67-1.58)$ & 0.925 & 24.65 & 17.70 & 1.39 & $(0.94-2.05)$ & 0.096 \\
\hline $6 B$ & 0.14 & 0.27 & 0.51 & $(0.31-0.83)$ & 0.007 & 0.63 & 0.54 & 1.15 & $(0.64-2.09)$ & 0.632 & 12.84 & 8.57 & 1.50 & $(0.90-2.49)$ & 0.116 \\
\hline $7 F$ & 3.29 & 3.18 & 1.03 & $(0.76-1.41)$ & 0.832 & 1.80 & 1.42 & 1.26 & (0.93- & 0.133 & 8.93 & 8.06 & 1.11 & 1.49) & 0.484 \\
\hline $9 \mathrm{~V}$ & 2.38 & 2.57 & 0.93 & $(0.62-1.40)$ & 0.715 & 0.87 & .75 & 1.16 & $(0.8$ & 0.426 & 7.85 & 5.98 & 1.13 & $(0.80$ & 0.493 \\
\hline 14 & 3.93 & 4.29 & 0.92 & $(0.53-1.58)$ & 0.752 & 33 & 73 & 0.85 & $(0.5$ & 98 & 51 & 10.31 & 1.12 & 70) & 0.607 \\
\hline $18 \mathrm{C}$ & 2.33 & 2.10 & 1.11 & $(0.77-1.60)$ & 0.578 & 0.55 & 0.43 & 1.27 & $(0.89-1.83)$ & 0.190 & 4.65 & 4.30 & 1.08 & $(0.77-1.52)$ & 0.652 \\
\hline $19 \mathrm{~A}$ & 0.47 & 1.61 & 0.29 & $(0.18-0.46)$ & $<0.001$ & 0.39 & 0.51 & 0.76 & $(0.44-1.32)$ & 0.319 & 7.72 & 6.16 & 1.25 & $(0.77-2.03)$ & 0.354 \\
\hline $19 F$ & 4.15 & 5.78 & 0.72 & $(0.49-1.06)$ & 0.100 & 1.72 & 1.96 & 0.88 & $(0.55-1.43)$ & 0.589 & 9.37 & 10.77 & 0.87 & $(0.56-1.34)$ & 0.524 \\
\hline $23 \mathrm{~F}$ & 0.59 & 0.87 & 0.68 & $(0.42-1.12)$ & 0.128 & 0.63 & 0.46 & 1.37 & $(0.91-2.07)$ & 0.139 & 13.80 & 9.60 & 1.44 & $(0.97-2.12)$ & 0.069 \\
\hline
\end{tabular}

$\mathrm{Cl}$, confidence interval.

a) $95 \% \mathrm{Cls}$ were calculated as the anti-log of the $\mathrm{Cl}$ of the mean difference of log-transformed geometric mean concentration. ${ }^{\text {b) }} \mathrm{P}$ value for the mean difference was determined using the 2 -sample $t$ test.

Boldface indicates a statistically significant difference with $P<0.05$.

Table 4. Proportion of subjects who achieved a multiplex opsonophagocytic assay titer of $\geq 1: 8$

\begin{tabular}{|c|c|c|c|c|c|c|c|c|c|c|}
\hline \multirow[b]{2}{*}{ Serotype } & \multicolumn{5}{|c|}{ After the primary dose } & \multicolumn{5}{|c|}{ After the booster dose } \\
\hline & $\begin{array}{c}\text { GBP411 } \\
(n=15)\end{array}$ & $\begin{array}{l}\text { Prevnar } 13 \\
(\mathrm{n}=51)\end{array}$ & Difference & $95 \% \mathrm{Cl}^{\mathrm{a})}$ & $P$ value $^{\text {b) }}$ & $\begin{array}{c}\text { GBP411 } \\
(n=15)\end{array}$ & $\begin{array}{l}\text { Prevnar } 13 \\
\quad(n=15)\end{array}$ & Difference & $95 \% \mathrm{Cl}^{\mathrm{a})}$ & $P$ value ${ }^{\text {b) }}$ \\
\hline 1 & 73.33 & 100.00 & -26.67 & $(-49.05$ to -4.29$)$ & $0.100^{c)}$ & 100 & 100 & 0.00 & $(0.00-0.00)$ & - \\
\hline 4 & 100.00 & 100.00 & 0.00 & $(0.00-0.00)$ & - & 100 & 100 & 0.00 & $(0.00-0.00)$ & - \\
\hline 5 & 100.00 & 100.00 & 0.00 & $(0.00-0.00)$ & - & 100 & 100 & 0.00 & $(0.00-0.00)$ & - \\
\hline $6 \mathrm{~A}$ & 100.00 & 100.00 & 0.00 & $(0.00-0.00)$ & - & 100 & 100 & 0.00 & $(0.00-0.00)$ & - \\
\hline $6 \mathrm{~B}$ & 86.67 & 93.33 & -6.67 & $(-28.00$ to 14.67$)$ & $1.000^{c)}$ & 100 & 100 & 0.00 & $(0.00-0.00)$ & - \\
\hline $7 F$ & 100.00 & 100.00 & 0.00 & $(0.00-0.00)$ & - & 100 & 100 & 0.00 & $(0.00-0.00)$ & - \\
\hline $9 \mathrm{~V}$ & 100.00 & 100.00 & 0.00 & $(0.00-0.00)$ & - & 100 & 100 & 0.00 & $(0.00-0.00)$ & - \\
\hline 14 & 100.00 & 100.00 & 0.00 & $(0.00-0.00)$ & - & 100 & 100 & 0.00 & $(0.00-0.00)$ & - \\
\hline $18 \mathrm{C}$ & 100.00 & 100.00 & 0.00 & $(0.00-0.00)$ & - & 100 & 100 & 0.00 & $(0.00-0.00)$ & - \\
\hline $19 A$ & 100.00 & 100.00 & 0.00 & $(0.00-0.00)$ & - & 100 & 100 & 0.00 & $(0.00-0.00)$ & - \\
\hline $19 F$ & 100.00 & 100.00 & 0.00 & $(0.00-0.00)$ & - & 100 & 100 & 0.00 & $(0.00-0.00)$ & - \\
\hline $23 \mathrm{~F}$ & 93.33 & 93.33 & 0.00 & $(-17.85$ to 17.85$)$ & $1.000^{c)}$ & 100 & 100 & 0.00 & $(0.00-0.00)$ & - \\
\hline
\end{tabular}

$\mathrm{Cl}$, confidence interval.

a) $95 \% \mathrm{Cl}$ for the difference in proportions between the treatment groups were determined using the Wald method. ${ }^{\text {b) }} \mathrm{P}$ value for the proportion difference was determined using ${ }^{\mathrm{c}}$ Fisher exact test.

it was lower for serotype 7F in the comparator vaccine group $(P=0.027)$. However, after the booster dose, the GMT increased markedly from after the primary doses for all the 12 serotypes (Table 5). Intergroup differences in GMTs with MOPA for all the 12 serotypes were not significant.

\section{Safety results}

The incidence rate of solicited local and systemic AEs in the GBP411 and comparator vaccine are shown in Table 6. A majority of the subjects experienced at least one solicited local and systemic $\mathrm{AE}$ within 7 days of vaccination throughout the study. The overall incidence of solicited local AEs was lower in the GBP411 group than in the comparator vaccine group; however, the intergroup difference was not significant $(77.55 \%$ and $90.20 \%$ in the GBP411 and comparator vaccine groups, respectively, $P=0.085$ ). There was no difference in the inci- dence of fever $>38.5^{\circ} \mathrm{C}$ (GBP411: $16.33 \%$ vs. comparator: 15.69\%). The overall incidence of solicited systemic AEs was significantly lower in the GBP411 group than in the comparator vaccine group $(79.59 \%$ vs. $98.04 \%, P=0.003)$. With regard to the individual solicited local or systemic AEs, no significant difference was noted between the groups except that the incidence of pain/tenderness (local AE) and irritability (systemic AE) was significantly lower in the GBP411 group $(P=0.042$ and $P=0.012$, respectively).

Unsolicited AEs were experienced by 38 subjects each in the GBP411 group (77.55\%, 115 events) and the comparator vaccine group (74.51\%, 101 events), and the intergroup differences were not significant $(P=0.722)$. The majority of unsolicited AEs were mild or moderate in severity. The most commonly reported System Organ Class was infections and infestations (59.18\% in the GBP411 group and $64.71 \%$ in the comparator 
Table 5. Geometric mean titer in the multiplex opsonophagocytic assay

\begin{tabular}{|c|c|c|c|c|c|c|c|c|c|c|}
\hline \multirow[b]{2}{*}{ Serotype } & \multicolumn{5}{|c|}{ After the primary dose } & \multicolumn{5}{|c|}{ After the booster dose } \\
\hline & $\begin{array}{c}\text { GBP411 } \\
(n=15)\end{array}$ & $\begin{array}{c}\text { Prevnar } 13 \\
(n=51)\end{array}$ & Ratio & $95 \% \mathrm{Cl}^{\mathrm{a})}$ & $P$ value $^{\text {b) }}$ & $\begin{array}{c}\text { GBP411 } \\
(n=15)\end{array}$ & $\begin{array}{l}\text { Prevnar } 13 \\
(n=15)\end{array}$ & Ratio & $95 \% \mathrm{Cl}^{\mathrm{a})}$ & $P$ value ${ }^{\mathrm{b})}$ \\
\hline 1 & 33.41 & 82.60 & 0.40 & $(0.15-1.10)$ & 0.077 & 306.86 & 335.01 & 0.92 & $(0.35-2.46)$ & 0.856 \\
\hline 4 & 1433.27 & 1624.98 & 0.88 & $(0.49-1.60)$ & 0.667 & 2356.75 & 2012.59 & 1.17 & $(0.62-2.23)$ & 0.620 \\
\hline 5 & 247.97 & 476.18 & 0.52 & $(0.28-0.97)$ & 0.040 & 1029.07 & 781.61 & 1.32 & $(0.60-2.93)$ & 0.486 \\
\hline $6 \mathrm{~A}$ & 1587.97 & 1302.79 & 1.22 & $(0.64-2.34)$ & 0.537 & 5221.01 & 2960.63 & 1.76 & $(0.62-5.05)$ & 0.284 \\
\hline $6 \mathrm{~B}$ & 335.61 & 208.34 & 1.61 & $(0.32-8.19)$ & 0.553 & 4193.49 & 2978.61 & 1.41 & $(0.69-2.90)$ & 0.340 \\
\hline $7 F$ & 6420.08 & 3735.14 & 1.72 & $(1.07-2.77)$ & 0.027 & 9356.14 & 6022.41 & 1.55 & $(0.88-2.76)$ & 0.127 \\
\hline $9 \mathrm{~V}$ & 417.17 & 249.40 & 1.67 & $(0.71-3.95)$ & 0.230 & 1612.58 & 790.63 & 2.04 & $(0.85-4.91)$ & 0.107 \\
\hline 14 & 1355.34 & 624.20 & 2.17 & $(0.50-9.56)$ & 0.293 & 3631.91 & 2210.78 & 1.64 & $(0.74-3.66)$ & 0.213 \\
\hline $18 C$ & 1184.10 & 1121.06 & 1.06 & $(0.63-1.80)$ & 0.834 & 1847.12 & 1416.74 & 1.30 & $(0.73-2.35)$ & 0.361 \\
\hline $19 A$ & 354.18 & 586.53 & 0.60 & $(0.27-1.37)$ & 0.214 & 5400.34 & 3853.18 & 1.40 & $(0.72-2.77)$ & 0.318 \\
\hline $19 F$ & 531.65 & 547.12 & 0.97 & $(0.44-2.19)$ & 0.943 & 1836.58 & 2011.74 & 0.91 & $(0.42-1.99)$ & 0.813 \\
\hline $23 F$ & 403.25 & 551.82 & 0.73 & $(0.14-3.94)$ & 0.706 & 5231.58 & 6213.96 & 0.84 & $(0.39-1.83)$ & 0.651 \\
\hline
\end{tabular}

$\mathrm{Cl}$, confidence interval.

a) $95 \% \mathrm{Cl}$ was calculated as the anti-log of the $\mathrm{Cl}$ of the mean difference of log-transformed geometric mean titer. ${ }^{\text {b) }} \mathrm{P}$ value for the mean difference was determined using the 2 -sample $t$ test.

Boldface indicates a statistically significant difference with $P<0.05$.

Table 6. Incidence rates of solicited local and systemic AEs

\begin{tabular}{lcccc}
\hline \multirow{2}{*}{ Variable } & \multicolumn{4}{c}{ GBP411 vs. Prevnar 13 } \\
\cline { 2 - 5 } & $\begin{array}{c}\text { GBP411 } \\
(\mathrm{n}=49)\end{array}$ & $\begin{array}{c}\text { Prevnar 13 } \\
(\mathrm{n}=51)\end{array}$ & $95 \% \mathrm{Cl}^{\mathrm{a})}$ & $P$ value $^{\mathrm{b})}$ \\
\hline Solicited local AEs & 77.55 & 90.20 & $(75.32-90.57)$ & 0.085 \\
$\quad$ Pain/tenderness & 69.39 & 86.27 & $(68.61-85.67)$ & 0.042 \\
Erythema/redness & 36.73 & 45.10 & $(31.26-51.29)$ & 0.395 \\
Induration/swelling & 30.61 & 25.49 & $(19.48-37.87)$ & 0.569 \\
Solicited systemic AEs & 79.59 & 98.04 & $(81.17-94.38)$ & 0.003 \\
$\quad$ Feverc) & 40.82 & 50.98 & $(35.98-56.26)$ & 0.308 \\
$\quad$ Mild & 32.65 & 37.25 & - & - \\
$\quad$ Moderate & 10.20 & 11.76 & - & - \\
$\quad$ Severe & 6.12 & 3.92 & - & - \\
Vomiting & 30.61 & 17.65 & $(16.02-33.57)$ & 0.129 \\
Diarrhea & 36.73 & 25.49 & $(22.13-41.03)$ & 0.224 \\
Irritability & 67.35 & 88.24 & $(68.61-85.67)$ & 0.012 \\
Sleepiness & 63.27 & 76.47 & $(60.02-78.76)$ & 0.150 \\
Decreased appetite & 34.69 & 27.45 & $(22.13-41.03)$ & 0.434 \\
\hline
\end{tabular}

$\mathrm{AE}$, adverse event; $\mathrm{Cl}$, confidence interval.

a) $95 \%$ Clopper-Pearson $\mathrm{Cl}$ was calculated with binomial distribution. ${ }^{\text {b) }} \mathrm{P}$ values were determined using the chi-square test. ${ }^{\mathrm{c}}$ Fever $\left({ }^{\circ} \mathrm{C}\right)$ : mild $(38.0-$ 38.4), moderate (38.5-38.9), and severe (39.0-40.0).

vaccine group), which was contributed to by the high incidence of various respiratory infections. Thirty serious AEs, mostly infections and infestations, were experienced by 11 subjects (22.45\%) in the GBP411 group and 7 subjects (13.73\%) in the comparator vaccine group. All of these serious AEs were severe in intensity and unrelated to the study vaccine, and fully resolved.

\section{Discussion}

After the primary doses, GBP411 was considered to induce the immune response well given that the proportion of subjects with pneumococcal serotype-specific IgG antibody concentrations of $\geq 0.35 \mu \mathrm{g} / \mathrm{mL}$ ranged from $85.42 \%$ to $100.00 \%$, other than those for serotypes $6 \mathrm{~B}, 19 \mathrm{~A}$, and $23 \mathrm{~F}$. Relatively low immunogenicity for serotypes $6 \mathrm{~B}$ and $23 \mathrm{~F}$ with a dosing schedule of 2 primary doses has been reported previously. ${ }^{5,9)}$ According to the results from the previous clinical trials conducted with Prevnar 13 in $2 p+1$ schedule, the proportion of infants achieving an antibody level of $>0.35 \mu \mathrm{g} / \mathrm{mL}$ ranged from $27.9 \%$ to $58.4 \%$ for $6 \mathrm{~B}$ and from $55.8 \%$ to $68.6 \%$ for $23 \mathrm{~F} .{ }^{10)}$ Compared to these results, low immunogenicity of GBP411 for these 2 serotypes (6B: $20.83 \%, 23 \mathrm{~F}: 70.83 \%$ ) in this study is unremarkable and the comparator vaccine's response against serotype $6 \mathrm{~B}$ was low as $39.22 \%$. In addition, the serotype-specific correlate of protection was $0.16 \mu \mathrm{g} / \mathrm{mL}$ in a cohort study conducted in the United Kingdom, ${ }^{11)}$ suggesting that high antibody levels are not needed to protect against IPD caused by serotype 6B. Of note, functional activity, measured by MOPA, was elicited by GBP411 for serotypes 6B, 19A, and 23F at similar percentages of subjects achieving 1:8 and comparable GMTs compared to the comparator vaccine both after primary and booster dose.

It is interesting that persistence of antibody until booster dose was notable for GBP411. The GMC for serotype 6A, 6B and 23F in GBP411 increased at pre-booster dose compared to that at post-primary dose (6A: $1.44 \mu \mathrm{g} / \mathrm{mL} \rightarrow 1.59 \mu \mathrm{g} / \mathrm{mL}$, 6B: $0.14 \mu \mathrm{g} / \mathrm{mL} \rightarrow 0.63 \mu \mathrm{g} / \mathrm{mL}, 23 \mathrm{~F}: 0.59 \mu \mathrm{g} / \mathrm{mL} \rightarrow 0.63 \mu \mathrm{g} /$ $\mathrm{mL}$ ), while the comparator vaccine increased only $6 \mathrm{~B}$ from $0.27 \mu \mathrm{g} / \mathrm{mL}$ to $0.54 \mu \mathrm{g} / \mathrm{mL}$, from previous studies, Prevnar 13 was already known to increase only $6 \mathrm{~B} .{ }^{10)}$ GBP411 elicited robust responses for all 12 serotypes after the booster dose, and specifically, postbooster responses (i.e., GMCs) for serotypes 6B, 19A, and 23F were higher with GBP411 than with the comparator vaccine, although this difference was not significant. As for the low immune response for serotype 19A after primary doses in GBP411, it was not able to find demographic difference 
between groups or any factors (i.e., concomitant medication that subject had or AEs that subject experienced) that could affect the immunogenicity of individual subjects. Further investigation will be needed through more studies, since the number of analyzed subjects was limited to around 50 in each group.

While this study was not designed to test the noninferiority of the study vaccine to the comparator vaccine, the history and guidelines of approval of other pneumococcal vaccines suggest that it is difficult and not necessary to confirm immunogenicity comparable to that of the comparator vaccine for all serotypes in PCV development. The WHO guideline ${ }^{12)}$ mentions additional considerations for functional antibodies and epidemiologic considerations are needed when the test vaccine does not meet the predefined criteria of noninferiority to the comparator vaccine for one or more serotypes. Thus, Prevnar 13 was approved for licensure by the US FDA and globally although it did not meet the criteria for noninferiority to Prevnar (a 7 valent PCV) for all serotypes. Further, despite the low immunogenicity of some serotypes after primary doses, European Medicines Agency approved $2 p+1$ regimen after confirming the robust immunogenicity after booster dose. ${ }^{10)}$

GBP411 showed a safety profile comparable to that of the comparator vaccine. The overall incidence of solicited systemic AEs was significantly lower in the GBP411 group than in the comparator vaccine group $(P=0.003)$. Particularly, the incidence of injection-site pain/tenderness (local $\mathrm{AE}$ ) and irritability (systemic $\mathrm{AE}$ ) was significantly lower in the GBP411 group than in the comparator vaccine group $(P<0.05)$. This result indicates that infants could potentially benefit from the lower incidence of adverse local and systemic reactions associated with GBP411 vaccination.

There were limitations in determining the immunogenic profile of GBP411 with this study, since there were only 100 subjects enrolled, especially MOPA was conducted in only 15 subjects in each group. Although this was an exploratory study to determine the immunogenicity and safety of GBP411, which is a 12 -valent PCV, the overall results indicate that compared to the comparator vaccine, GBP411 with a $2 p+1$ dosing schedule and the first dose administered at 6 to 10 weeks of age, induced a substantial immune response in ELISA and MOPA at the same time, and may be safe for administration to healthy infants. In addition, it will be necessary to confirm the immunogenicity and safety in various races and to observe the results of $3 p+0$ as well as $2 p+1$ regimen for the purpose of developing GBP411 to supply developing countries. More studies including a long-term immunogenicity follow-up study and pivotal study that will test the hypothesis, are needed to fully evaluate the immunogenicity and safety of GBP411 with a $2 p+1$ dosing schedule.

\section{Conflicts of interest}

No potential conflict of interest relevant to this article was reported.
Acknowledgments

This research was supported by the Bill and Melinda Gates Foundation (OPP1148601).

See the commentary "Pneumococcal conjugate vaccines: choice of schedule and product development" via https://doi. org/10.3345/cep.2019.01739.

\section{References}

1. Centers for Disease Control and Prevention. Global pneumococcal disease and vaccine [Internet]. Atlanta (GA): Centers for Disease Control and Prevention; 2020 [cited 2019 Mar 15]. Available from: https://www. cdc.gov/pneumococcal/global.html.

2. Wahl B, O'Brien KL, Greenbaum A, Majumder A, Liu L, Chu Y, et al. Burden of Streptococcus pneumoniae and Haemophilus influenzae type $b$ disease in children in the era of conjugate vaccines: global, regional, and national estimates for 2000-15. Lancet Glob Health 2018;6:e744-57.

3. Plotkin SA, Orenstein WA, Offit PA, Edwards KM. Plotkin's vaccines. 7th ed. Philadelphia (PA): Elsevier, 2018:774.

4. World Health Organization. Pneumococcus vaccines position paper. 2019 [Internet]. Geneva (Switzerland): World Health Organization; 2019 [cited 2019 Mar 15]. Available from: https://www.who.int/immunization/ policy/position_papers/pneumococcus/en/.

5. Rodgers GL, Esposito S, Principi N, Gutierrez-Brito M, Diez-Domingo J, Pollard AJ, et al. Immune response to 13-valent pneumococcal conjugate vaccine with a reduced dosing schedule. Vaccine 2013;31:4765-74.

6. Ladhani SN, Collins S, Djennad A, Sheppard CL, Borrow R, Fry NK, et al. Rapid increase in non-vaccine serotypes causing invasive pneumococcal disease in England and Wales, 2000-17: a prospective national observational cohort study. Lancet Infect Dis 2018;18:441-51.

7. Galanis I, Lindstrand A, Darenberg J, Browall S, Nannapaneni P, Sjöström $\mathrm{K}$, et al. Effects of PCV7 and PCV13 on invasive pneumococcal disease and carriage in Stockholm, Sweden. Eur Respir J 2016;47:1208-18.

8. U.S. Food \& Drug Administration. Toxicity grading scale for healthy adult and adolescent volunteers enrolled in preventive vaccine clinical trials [Internet]. Rockville (MD): U.S. Food \& Drug Administration; [cited 2019 Mar 15]. Available from: https://www.fda.gov/regulatory-information/ search-fda-guidance-documents/toxicity-grading-scale-healthy-adult-andadolescent-volunteers-enrolled-preventive-vaccine-clinical.

9. Scott P, Rutjes AW, Bermetz L, Robert N, Scott S, Lourenço T, et al. Comparing pneumococcal conjugate vaccine schedules based on 3 and 2 primary doses: systematic review and meta-analysis. Vaccine 2011;29: 9711-21.

10. European Medicines Agency. Prevnar 13, Procedure No. EMEA/H/C/ 001104 [Internet]. Amsterdam (The Netherlands): European Medicines Agency; c1995-2020 [cited 2018 Aug 30]. Available from https:/www. ema.europa.eu/en/medicines/human/EPAR/prevenar-13\#authorisationdetails-section.

11. Andrews NJ, Waight PA, Burbidge P, Pearce E, Roalfe L, Zancolli M, et al. Serotype-specific effectiveness and correlates of protection for the 13-valent pneumococcal conjugate vaccine: a postlicensure indirect cohort study. Lancet Infect Dis 2014;14:839-46.

12. World Health Organization. Annex 2: Guidelines on evaluation of similar biotherapeutic products (SBPs). WHO Technical Report Series No. 977 [Internet]. Geneva (Switzerland): World Health Organization; 2013 [cited 2018 Aug 30]. Available from: http://www.who.int/biologicals/publications/ trs/areas/biological_therapeutics/TRS_977_Annex_2.pdf?ua=1. 